\title{
On the critical forcing amplitude of forced nonlinear oscillators
}

\section{Communication}

\author{
Mariano Febbo ${ }^{1 *}$, Jinchen C. $\mathrm{Ji}^{2}$ \\ 1 Instituto de Física del Sur (CONICET) and Departamento de Física Universidad Nacional del Sur, \\ Avenida Alem 1253, 8000 Bahía Blanca, Argentina \\ 2 Faculty of Engineering and Information Technology, University of Technology, Sydney \\ Broadway NSW 2007, PO Box 123 Sydney, Australia
}

Received 12 March 2012; accepted 07 June 2013

\begin{abstract}
The steady-state response of forced single degree-of-freedom weakly nonlinear oscillators under primary resonance conditions can exhibit saddle-node bifurcations, jump and hysteresis phenomena, if the amplitude of the excitation exceeds a certain value. This critical value of excitation amplitude or critical forcing amplitude plays an important role in determining the occurrence of saddle-node bifurcations in the frequency-response curve. This work develops an alternative method to determine the critical forcing amplitude for single degree-of-freedom nonlinear oscillators. Based on Lagrange multipliers approach, the proposed method considers the calculation of the critical forcing amplitude as an optimization problem with constraints that are imposed by the existence of locations of vertical tangency. In comparison with the Gröbner basis method, the proposed approach is more straightforward and thus easy to apply for finding the critical forcing amplitude both analytically and numerically. Three examples are given to confirm the validity of the theoretical predictions. The first two present the analytical form for the critical forcing amplitude and the third one is an example of a numerically computed solution.

Keywords: Critical forcing amplitude • forced nonlinear systems• frequency-response curve

(c) Versita sp. z o.o.
\end{abstract}

\section{Introduction}

In single degree-of-freedom (SDOF) weakly nonlinear oscillators subjected to periodic excitations, nonlinear resonances may occur if the linearized natural frequency of the system and the frequency of an external excitation satisfy a certain relationship. A small-amplitude excitation may produce a relatively large-amplitude response under primary resonance conditions when the forcing frequency is

*E-mail: mfebbo@uns.edu.ar in the neighborhood of the linearized natural frequency. The critical threshold of the amplitude of excitation or, simply, the critical forcing amplitude is commonly referred to as a certain value of the excitation amplitude for which the response amplitude has one solution only if below this value and can have three solutions if above this value [13].

Beyond the critical forcing amplitude, the steady-state forced response of the nonlinear oscillators under primary resonance conditions may exhibit nonlinear dynamic behaviors including saddle-node bifurcations, jump and hysteresis phenomena [1-3]. Figure 1 shows a typical frequency-response curve of the amplitude of the response 
as a function of the external detuning, for a certain forcing amplitude above the critical value. There is one solution branch for the detuning in the regimes $\sigma<\sigma_{1}$ and $\sigma>\sigma_{2}$, and three coexisting solutions for $\sigma_{1}<\sigma<\sigma_{2}$, respectively. Two solution branches merge at points $A$ and $B$ where saddle-node bifurcations occur. It is noted that points $A$ and $B$ are the jump-up and jump-down points and the corresponding frequencies are called jumpup and jump-down frequencies because they are the frequencies where the frequency-response curve leads to a jump when the excitation frequency is swept from left-toright or right-to-left. These two points coincide with the locations of vertical tangency of the frequency-response curve. The values of detuning $\sigma_{1}, \sigma_{2}$ can be found based on this fact. A brief literature review is given here to

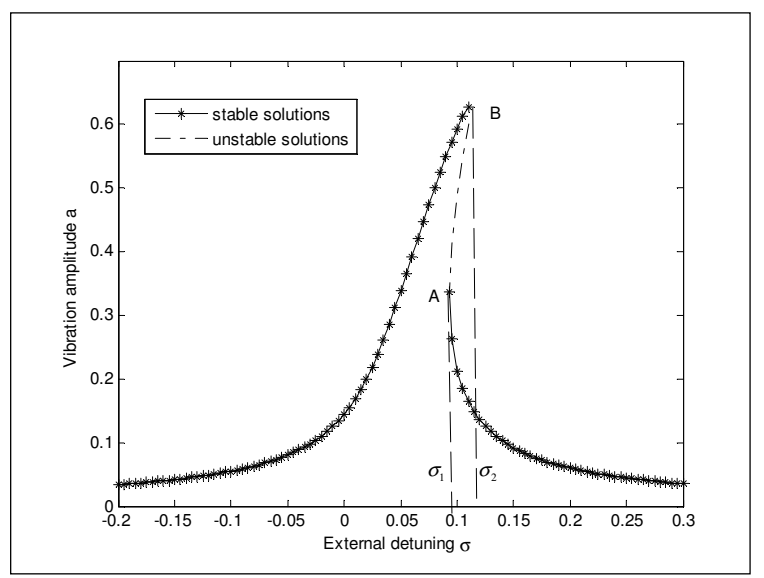

Figure 1. Typical frequency-response curve of the primary resonance response for certain forcing amplitude larger than its critical value. Solid lines denote stable solutions and dash-dot lines represent unstable solutions.

show the calculation of the critical forcing amplitude for SDOF nonlinear systems as well as for the jump frequencies. Kervorkian and Cole [4] used the method of multiple scales (MMS) to calculate the jump frequencies for a hardening nonlinear system. Friswell and Penny [1] and Worden [2] used the harmonic balance method (HBM) to calculate the jump frequencies of the Duffing oscillator with linear damping. Friswell and Penny employed a numerical approach to compute the jump frequencies while Worden used a different approach setting the discriminant of the frequency-response function equal to zero. Later, Malatkar and Nayfeh [3] developed a general procedure to determine the critical forcing amplitude and the jump frequencies based on the elimination theory of polynomials. To obtain their key results, they numerically solved a set of polynomial equations using an available software package.

This paper aims to present an alternative method to de- termine the critical forcing amplitude for forced nonlinear oscillators. The main advantage of the proposed method is that it does not require the calculation of the resultant or the Gröbner basis of two polynomials as employed for example by Malatkar and Nayfeh [3] in a previous approach to the same problem. Instead, the proposed method considers the calculation as an optimization problem with constraints, using the Lagrange multipliers approach. As a result, it is possible to find the critical forcing amplitude by computing the derivatives of two functions only. Three examples are given to show the effectiveness and validation of the proposed method. The first two give the critical forcing amplitude in analytical form while the last one computes the solution numerically.

To see the details of the calculation, we will analyze the simplest case of a weakly nonlinear damped oscillator subjected to periodic excitation having mass $m_{1}$, linear stiffness $k_{1}$, nonlinear stiffness $k_{3}$ and damping coefficient $c_{1}$, namely, the Duffing oscillator with periodic excitation. The equation of motion for the displacement amplitude $x$ can be readily found to be

$$
\ddot{x}+\mu_{10} \dot{x}+\omega_{10}^{2} x+\alpha x^{3}=f_{0} \cos (\omega t)
$$

where $\mu_{10}=c_{1} / m_{1}, \omega_{10}^{2}=k_{1} / m_{1}, \alpha=k_{3} / m_{1}$. For the case of primary resonances, the forcing frequency $\omega$ is assumed to be almost equal to $\omega_{10}$ and, therefore $\omega=\omega_{10}+\varepsilon \sigma$, where $\varepsilon$ is a small dimensionless parameter $\varepsilon \ll 1$ and $\sigma$ is an external detuning parameter.

The nonlinear oscillations of Eq. (1) have been extensively studied in the literature (for example $[5,6]$ ). By applying the MMS method the frequency-response equation for the amplitude of the first-order approximate solution can be obtained as

$$
\left(\frac{\mu_{10}}{2}\right)^{2} a^{2}+\left(-\sigma+g_{20} a^{2}\right)^{2} a^{2}=e^{2}
$$

where $a$ denotes the amplitude of the primary resonance response of the nonlinear system, $e=f_{0} /\left(2 \omega_{10}\right)$ and $g_{20}=3 \alpha /\left(8 \omega_{10}\right)$. For the brevity of explanation, $e$ will be referred to here as the amplitude of excitation, or simply forcing amplitude.

The locations of the jumps points (or the points of vertical tangency) are obtained by differentiating the amplitudefrequency relation (2) implicitly with respect to $a^{2}$ and then, setting $d \sigma / d a^{2}=0$. The resulting expression is,

$g(\sigma, a) \equiv\left(\frac{\mu_{10}}{2}\right)^{2}+2 a^{2}\left(-\sigma+g_{20} a^{2}\right) g_{20}+\left(-\sigma+g_{20} a^{2}\right)^{2}=0$

with solutions

$$
\sigma_{ \pm}=2 g_{20} a^{2} \pm \sqrt{g_{20}^{2} a^{4}-\left(\frac{\mu_{10}}{2}\right)^{2}}
$$


Determination of the critical forcing amplitude is to find a minimum value of forcing amplitude $e$ in Eq. (2) for which the amplitude $a$ has only one positive solution when the forcing amplitude is below its critical value and can have three positive solutions when the forcing amplitude is above its critical value. The frequency response curve for this critical forcing value of $e$ (will be denoted as $e_{c}$ ) has an inflection point at $\left(a_{c}, \sigma_{c}\right)$. The method to be developed in the following section provides the calculation of this minimum $e_{c}$ by taking into account the restriction imposed by the locations of vertical tangency.

\section{Mathematical formulation of the problem}

The problem of finding the critical amplitude of the excitation is a typical problem of minimizing a function subject to some prescribed constraints. To solve this, we recall some results of differential calculus applied to optimization problems (see for example [7]). In this case, the problem is summarized as finding the minimum of a differentiable function $f(\sigma, a) \equiv e^{2}$ (Eq. 2) subject to the constraints: $g(\sigma, a)=0$ (Eq. 3) and $h(\sigma, a) \equiv \frac{\partial \sigma}{\partial a^{2}}=0$. From the mathematical theory of optimization subject to constraints, we select the method of Lagrange multipliers to find the stationary points of function $f(\sigma, a)$ with these conditions. By applying to the present problem, this results in solving the following equation:

$$
d\left(f(\sigma, a)+\lambda_{1} g(\sigma, a)+\lambda_{2} h(\sigma, a)\right)=0
$$

where $d(r(\sigma, a))$ represents the total differential of function $r(\sigma, a)$ and $\lambda_{1}$ and $\lambda_{2}$ are the so-called Lagrange undetermined multipliers. The two equations obtained from Eq. (5) are

$$
\begin{aligned}
& \frac{\partial f}{\partial \sigma}+\lambda_{1} \frac{\partial g}{\partial \sigma}+\lambda_{2} \frac{\partial h}{\partial \sigma}=0 \\
& \frac{\partial f}{\partial a}+\lambda_{1} \frac{\partial g}{\partial a}+\lambda_{2} \frac{\partial h}{\partial a}=0
\end{aligned}
$$

which, together with the two constraint equations $g(\sigma, a)=0$ and $h(\sigma, a)=0$ give solutions for $\sigma, a, \lambda_{1}$ and $\lambda_{2}$. It is straightforward to obtain $\lambda_{2}$ from (6) which gives:

$$
\lambda_{2}=\left(-\frac{\partial f}{\partial \sigma}-\lambda_{1} \frac{\partial g}{\partial \sigma}\right) \frac{1}{\frac{\partial h}{\partial \sigma}}
$$

Substituting this value of $\lambda_{2}$ into Eq. (7) and using $\frac{\partial \sigma}{\partial a}=$ $2 a \frac{\partial \sigma}{\partial a^{2}}=2 a h(\sigma, a)$, we readily obtain:

$$
\frac{\partial f}{\partial a}+\lambda_{1} \frac{\partial g}{\partial a}+\left(-\frac{\partial f}{\partial \sigma}-\lambda_{1} \frac{\partial g}{\partial \sigma}\right) 2 a h(\sigma, a)=0
$$

From the fact that $h(\sigma, a)=0$, Eq. (9) becomes

$$
\frac{\partial f}{\partial a}+\lambda_{1} \frac{\partial g}{\partial a}=0
$$

Additionally, since $h(\sigma, a) \equiv \frac{\partial \sigma}{\partial a^{2}}=0 \Rightarrow \frac{\partial f}{\partial a} \equiv$ $2 a g(\sigma, a)=0$, then Eq. (10) can be simplified as:

$$
\frac{\partial g}{\partial a}=0
$$

This is an equation that provides $\sigma$ as a function of $a$ for the critical amplitude of excitation. After substituting this equation into $g(\sigma, a)=0$ we can obtain the corresponding response amplitude $a_{c}$ and the detuning $\sigma_{c}$ which provide the minimum value of $f(\sigma, a) \equiv e^{2}$ and consequently, the critical forcing amplitude $e_{c}$.

In summary, the detailed procedure for finding the critical forcing amplitude can be divided into three steps. The first step is to find the equation of determining the locations of the points of vertical tangency or jump frequencies by differentiating the frequency-response equation. For brevity, this is referred to here as the equation of vertical tangency, or Eq. (3) in this case. The second step is to obtain Eq. (11) by differentiating the equation of vertical tangency with respect to the response amplitude $a$. The third step is to solve the response amplitude $a$ and external detuning $\sigma$ (or frequency $\omega$ ) from these two equations and then substitute them into the frequency-response equation to obtain the critical forcing amplitude $e_{c}$. For such simple problems as will be discussed in the first two examples, the critical forcing amplitude can be obtained in explicit expressions.

\subsection{Examples and comparison with the exist- ing methods}

Here, we consider three examples for the calculation of the critical forcing amplitude. The first one is the Duffing oscillator subjected to periodic excitation which was analyzed in Section 1. The second one is a quasi-zero stiffness oscillator under inertial excitation [8] used as a nonlinear ultra-low frequency vibration isolator and the third one is a finite extensibility nonlinear oscillator [9] commonly used to model the impossibility of real oscillators to extend to infinity. In the first two cases, the values of the critical forcing amplitude are possible to be found analytically. Instead, the last case is an example of the application of the proposed method where the critical forcing amplitude is numerically obtained. 


\subsubsection{Duffing equation with periodic excitation}

Let us return to the case considered in Section 1. This time, we explicitly develop the solution for the critical forcing amplitude $e_{c}$ resulting from Eq. (1). Letting $\frac{\partial g}{\partial a}=0$ results in $\sigma=\frac{3}{2} g_{20} a^{2}$. Then substituting it into $g(\sigma, a)=$ 0 we obtain the following expression for $a^{2}$ :

$$
a^{2}=\sqrt{\frac{1}{3}} \frac{\mu_{10}}{g_{20}}
$$

The final expression for $e_{c}$ is obtained by substituting this value of $a^{2}$ into Eq. (2):

$$
e_{c}=\sqrt{\frac{1}{3 \sqrt{3}} \frac{\mu_{10}^{3}}{g_{20}}}
$$

Fig. 2 shows two frequency-response curves for various values of $e$. One is with the critical forcing amplitude $e_{c}$, and the other is with a slightly different value of $e=e_{u}=$ $\sqrt{\frac{\mu_{10}^{3}}{4 g_{20}}}$ obtained from [10] (relative error between $e_{c}$ and $e_{u}$ is $\left.\Delta_{e}(\%) \approx 14 \%\right)$ plotted for comparison. In all cases we use $g_{20}=0.0375$ and $\mu_{10}=1$. The validity of the obtained $e_{c}$ is evident.

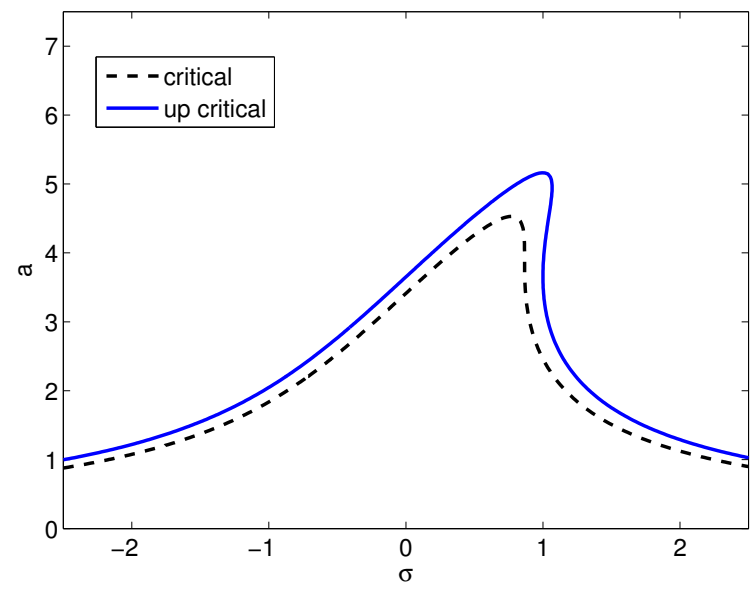

Figure 2. Different frequency-response curves to show the critical forcing amplitude for the Duffing oscillator as calculated using the proposed method (dotted line) and a slightly larger value than this (solid line) extracted from [10]. Data plotted using $g_{20}=0.0375, \mu_{10}=1$. In this case the critical forcing amplitude can be analytically obtained (see text).

\subsubsection{Critical forcing amplitude using Gröbner basis method}

In this subsection, we compare our method for the calculation of the critical forcing amplitude with another method borrowed from [3]. In that work, Malatkar and Nayfeh proposed essentially two methods to obtain the critical forcing amplitude. The first method is based on the Sylvester resultant while the second one uses the Gröbner basis for polynomials. We arbitrarily select the second one based on the Gröbner basis to perform a comparison between our proposed approach and their method. The Gröbner basis method uses the fact that $f^{\prime}(\sigma, a)$ and $f^{\prime \prime}(\sigma, a)$, which are the first and the second derivative of $f(\sigma, a)$ with respect to $a$ respectively, vanish at the inflection point of coordinates $\left(\sigma_{c}, a_{c}\right)$. The method can be divided into three steps, namely

- Calculate the first two derivatives of the frequencyresponse equation $f^{\prime}(\sigma, a)$ and $f^{\prime \prime}(\sigma, a)$.

- Compute the Gröbner basis for the polynomials $f^{\prime}(\sigma, a)$ and $f^{\prime \prime}(\sigma, a)$ and obtain two polynomials $G_{1}, G_{2}$ which also vanish at the inflection point $\left(\sigma_{c}, a_{c}\right)$.

- Obtain $a_{c}$ from $G_{1}=0$ and $\sigma_{c}$ from $G_{2}=0$. Finally, replace those values of $a_{c}$ and $\sigma_{c}$ into the frequency-response curve Eq. (2) to obtain $e_{c}$.

For the example discussed in Section 2.1.1, from Eq. (2) we can obtain the first two derivatives of the frequencyresponse equation as

$$
\begin{gathered}
f^{\prime}(\sigma, a)=\frac{\mu_{10}}{2} a+6 a^{5} g_{20}-8 a^{3} g_{20} \sigma+2 a \sigma^{2} \\
f^{\prime \prime}(\sigma, a)=\frac{\mu_{10}^{2}}{2}+30 a^{4} g_{20}^{2}-24 a^{2} g_{20} \sigma+2 \sigma^{2}
\end{gathered}
$$

To compute the Gröbner basis for polynomials $f^{\prime}(\sigma, a)$ and $f^{\prime \prime}(\sigma, a)$ we use the Groebner Basis function of Maple to obtain the following results:

$G_{1}(\sigma, a)=-a^{3} \mu_{10}^{2}+3 g_{20}^{2} a^{7} ; G_{12}(\sigma, a)=-3 a^{5} g_{20}+2 a^{3} \sigma$

Equating $G_{1}=0$ we obtain $a^{2}=\sqrt{\frac{1}{3}} \frac{\mu_{10}}{g_{20}}$ which is the same as Eq. (12) obtained using our proposed method, and then from $G_{2}=0$ we have $\sigma=\frac{3}{2} g_{20} a^{2}$ which has been obtained from making $\frac{\partial g}{\partial a}=0$ in Section 2.1.1. With these two values of $\sigma$ and $a$, replacing them into Eq. (2) we obtain

$$
e_{c}=\sqrt{\frac{1}{3 \sqrt{3}} \frac{\mu_{10}^{3}}{g_{20}}}
$$

which is the same value obtained from our method (see Eq. 16). As it can be seen from the beginning, the existing method is based on calculating the Groebner basis from a set of given polynomials which is not a trivial matter. Instead, our proposed method is based on finding a derivative, which is more straightforward. 


\subsubsection{Cubic spring with inertial excitation}

This type of quasi-zero stiffness SDOF nonlinear oscillator has been studied by [8] and [11] and is possible to be found in practical applications of nonlinear absorbers. The equation of motion of such a system with inertial excitation is:

$$
\ddot{x}+2 \xi \dot{x}+\gamma x^{3}=-\omega^{2} f \cos (\omega t)
$$

where $\xi$ is the damping coefficient and $\gamma$ is a nonlinear parameter that measures the degree of the nonlinearity. After applying, for example, the HBM and considering $x=$ $a \cos (\omega t)$ we arrive at the frequency-amplitude relation:

$$
\frac{9}{16} \gamma^{2} \frac{a^{6}}{\omega^{4}}-\frac{3}{2} \gamma \frac{a^{4}}{\omega^{2}}+\left(1+4 \frac{\xi^{2}}{\omega^{2}}\right) a^{2}=e^{2}
$$

where we have set $f=e$ for the sake of consistency of discussion.

Then, the function $g$ is calculated by differentiating Eq.(18) with respect to $a^{2}$ and setting $d \omega / d a^{2}=0$. It results in:

$$
g=\frac{27}{16} \gamma^{2} \frac{a^{4}}{\omega^{4}}-3 \gamma \frac{a^{2}}{\omega^{2}}+1+4 \frac{\xi^{2}}{\omega^{2}}
$$

Following the proposed method, making $\frac{\partial g}{\partial a}=0$ leads us to $\omega^{2}=\frac{27}{24} \gamma a^{2}$. Then, substituting it into $g(\omega, a)=0$ we obtain $a^{2}$ as:

$$
a^{2}=\frac{32}{3} \frac{\xi^{2}}{\gamma}
$$

Then, the resulting expression for the critical forcing amplitude $e_{c}$ is obtained by substituting this value of $a^{2}$ into Eq. (18):

$$
e_{c}=\sqrt{\frac{128}{27 \gamma}} \xi
$$

Figure 3 shows the frequency response curves for $e_{c}$ given by Eq. (21) and for $e_{u}=\sqrt{\frac{8}{9} \frac{(1+\sqrt{3})^{2}(5+3 \sqrt{3})}{\gamma(2+\sqrt{3})^{2}}} \xi$ calculated from [10] for comparison. We use $\gamma=3.3 \times 10^{-3}, \xi=0.04$ for all cases. Again, the validity of the method developed is evident.

\subsubsection{Finite extensibility oscillator with periodic excita- tion}

As a final example we analyze the case of a finite extensibility nonlinear oscillator. This type of systems is widely used to model real oscillators which can not be extended to infinity. For example, such systems can be found in the literature when modelling the bonds between molecules in a polymer or DNA molecule [12]. Unlike the previous two cases, we select this system as an example of a numerically obtained $e_{c}$. The modulation equation, which is

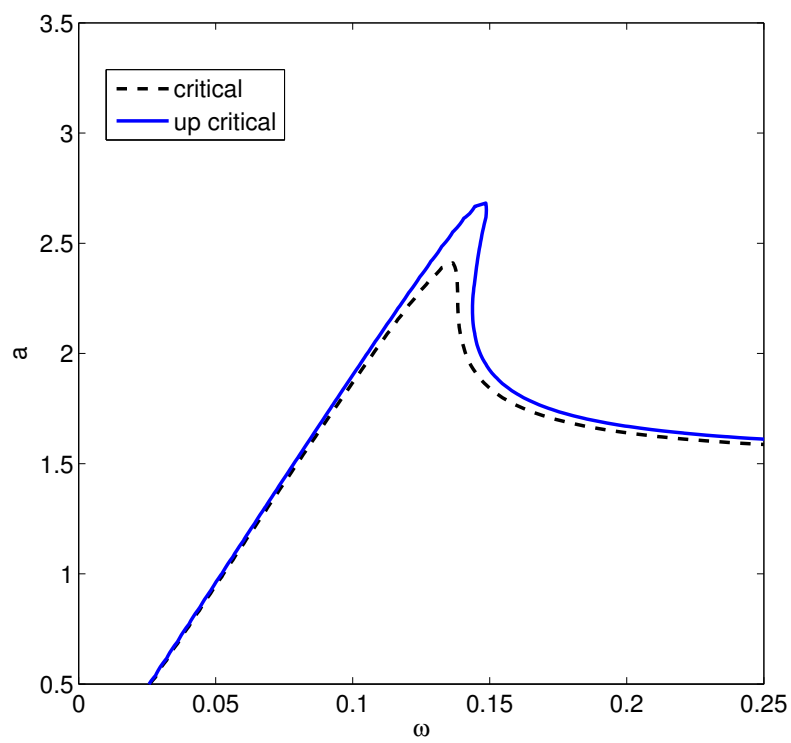

Figure 3. Frequency-response curves to show the critical forcing amplitude for the quasi-zero stiffness oscillator as calculated using the proposed method (dotted line) and a slightly larger value than this (solid line) extracted from [10]. Data plotted using $\gamma=3.3 \times 10^{-3}, \xi=0.04$. In this case the critical forcing amplitude can be analytically obtained (see text).

the result of applying the HBM to the equation of motion of this type oscillator is given in [9] and reproduced here to be:

$$
a^{2}\left(\frac{2 \omega_{N L}^{2}}{\sqrt{1-a^{2}}+1-a^{2}}-\omega^{2}\right)^{2}+4 \xi^{2} \omega_{N L}^{2} \omega^{2} a^{2}=e^{2}
$$

where, $a$, $e$ and $\xi$ have the same meaning as above and $\omega_{N L}$ is a nonlinear parameter. Then, function $g$ is obtained from differentiating Eq. (22) implicitly with respect to $a^{2}$ and setting $d \omega / d a^{2}=0$. The resulting expression is:

$$
\begin{aligned}
g \equiv( & \left.\frac{2 \omega_{N L}^{2}}{\sqrt{1-a^{2}}+1-a^{2}}-\omega^{2}\right)^{2} \\
+ & 2 a^{2} \omega_{N L}^{2}\left(\frac{2 \omega_{N L}^{2}}{\sqrt{1-a^{2}}+1-a^{2}}-\omega^{2}\right) \\
& \frac{\left(2+\left(1-a^{2}\right)^{-1 / 2}\right)}{\left(\sqrt{1-a^{2}}+1-a^{2}\right)^{2}}+4 \xi^{2} \omega_{N L}^{2} \omega^{2}=0
\end{aligned}
$$

where the value of $\omega$ can be found to be

$$
\omega_{ \pm}=\omega_{N L} \sqrt{b\left(a^{2}, \xi^{2}\right) \pm \sqrt{b^{2}\left(a^{2}, \xi^{2}\right)-4 c\left(a^{2}\right)}}
$$


where

$b\left(a^{2}, \xi^{2}\right)=\frac{2}{\sqrt{1-a^{2}}+1-a^{2}}+\frac{a^{2}\left(2+\left(1-a^{2}\right)^{-1 / 2}\right)}{\left(\sqrt{1-a^{2}}+1-a^{2}\right)^{2}}-2 \xi^{2}$

and

$$
c\left(a^{2}\right)=\frac{\left(\left(1-a^{2}\right)^{-1 / 2}+1+a^{2}\right)}{\left(\sqrt{1-a^{2}}+1-a^{2}\right)^{3}}
$$

Then, combining Eqs. (23) and (24) the function $g$ is simply written as

$$
g=\omega_{ \pm} \mp \omega_{N L} \sqrt{b\left(a^{2}, \xi^{2}\right)-\sqrt{b^{2}\left(a^{2}, \xi^{2}\right)-4 c\left(a^{2}\right)}}=0
$$

Due to the complicate expression for function $g$, an explicit expression for the critical forcing amplitude cannot be given. Instead, a numerical solution will be sought. After making $\frac{\partial g}{\partial a}=0$ and solving it for $a$ (numerically) we then substitute this value of $a=a_{c}$ into Eq. (24) for $\omega_{-}$to obtain $\omega_{c}$. Note that we have picked the value of $\omega_{-}$for $\omega$. If instead we have taken the value of $\omega_{+}$this would result in obtaining a complex value of $a$ which makes no sense. Finally, we substitute this pair $\left(\omega_{c}, a_{c}\right)$ into Eq. (22) to obtain $e_{c}$. The obtained frequency-response curve for $e_{c}$ together with another curve calculated using the value $e$ extracted from [10] are shown in Fig. 4 for $\xi=0.2$ and $\omega_{N L}=0.75$. Again, this numerically obtained value of $e_{c}$ is as expected.

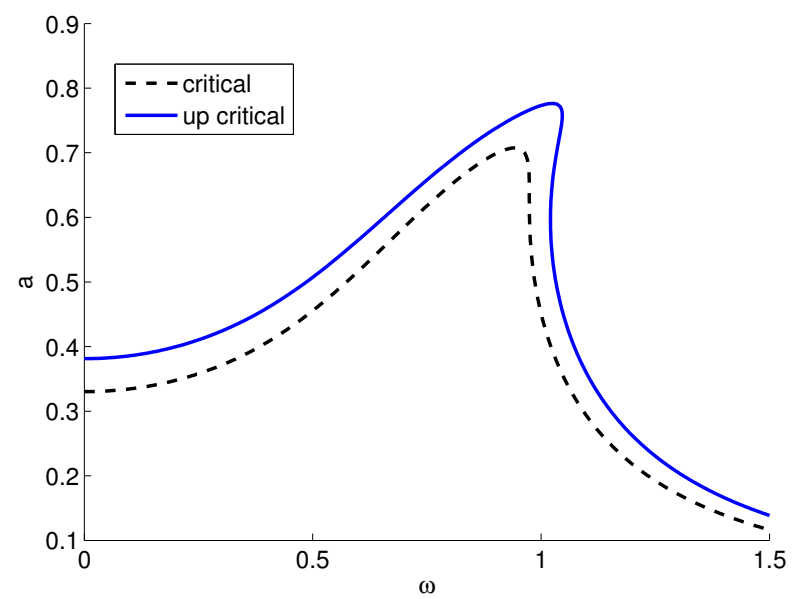

Figure 4. Frequency-response curves to show the critical forcing amplitude for the finite extensibility nonlinear oscillator as calculated using proposed method (dotted line) and a slightly larger value than this (solid line) extracted from [10]. Data plotted using $\xi=0.2$ and $\omega_{N L}=0.75$. In this case the critical forcing amplitude is numerically computed (see text).

\section{Conclusion}

An alternative method to obtain the critical forcing amplitude for SDOF nonlinear oscillators has been proposed in this work. The proposed method involves in setting the problem into an optimization problem with constraints, imposed by the existence of locations of vertical tangency and then using Lagrange multipliers approach to solve it. Unlike previous approaches to the same problem, which are based on the calculation of the Sylvester resultant or the Gröbner basis of polynomials, the main advantage of the proposed method is that it is easy to apply since it requires only the computation of the derivative of two functions. Briefly, the procedure for applying the proposed method includes differentiating the equation of vertical tangency to obtain another equation, solving these two equations to obtain the response amplitude and detuning (or frequency) and substituting the resultant response amplitude and detuning into the frequency-response equation to obtain the critical forcing amplitude. Three examples were given to confirm the validity of the proposed method that was applied to obtain the critical forcing amplitude in both analytical and numerical scenarios.

\section{Acknowledgments}

M. Febbo acknowledges financial support from CONICET and from Secretaría General de Ciencia y Tecnología of Universidad Nacional del Sur at the Departmento de Física ( PGI 24F/050).

\section{References}

[1] Friswell M.I., Penny J.E.T., The accuracy of jump frequencies in series solutions of the response of a Duffing oscillator, J SOUND VIB., 1994,169,261-269.

[2] Worden K., On jump frequencies in the response of the Duffing oscillator, J SOUND VIB., 1996,198,522525.

[3] Malatkar P., Nayfeh A. H., Calculation of the jump frequencies in the response of s.d.o.f. non-linear systems, J SOUND VIB., 2002,254,1005-1011.

[4] Kevorkian J., Cole J. D., Perturbation Methods in Applied Mathematics, Springer, New York, 1981.

[5] Nayfeh A.H., Mook D.T., Nonlinear Oscillations, John Wiley and Sons, New York, USA, 1979.

[6] Stoker J.J., Nonlinear Vibrations, Interscience, New York, USA, 1950.

[7] Riley K.F., Hobson M.P., Bence S.J., Mathematical Methods for Physics and Engineering, Cambridge 
University Press, Cambridge, UK, 2002.

[8] Alabuzhev P., Gritchin A., Kim L., Migirenko G., Chon V., Stepanov P., Vibration Protecting and Measuring Systems with Quasi- Zero Stiffness, Hemisphere Publishing, New York, USA, 1989.

[9] Febbo M., Harmonic response of a class of finite extensibility nonlinear oscillators, PHYS SCRIPTA., 2011,83,1-12.

[10] Ji J.C., Zhang N., Suppression of the primary resonance vibrations of a forced nonlinear system us- ing a dynamic vibration absorber, J SOUND VIB., 2010,329,2044-2056.

[11] Ibrahim R. A., Recent advances in nonlinear passive vibration isolators, J SOUND VIB., 2008,314,371452.

[12] Cheon M., Chang I., Koplik J., Banavar J. R., Chain molecule deformation in a uniform flow. A computer experiment, EUROPHYS LETT., 2002,58,215-221. 